\title{
A second hit somatic (p.R905W) and a novel germline intron-mutation of TSC2 gene is found in intestinal lymphangioleiomyomatosis: a case report with literature review
}

Bogyeong Han ${ }^{1}$, Juhwan Lee ${ }^{2}$, Yoon Jin Kwak', Hyun-Young Kim ${ }^{3}$ Kwang Hoon Lee ${ }^{4}$, Yumi Shim ${ }^{4}$ Hyunju Lee $2^{2^{*}}$ and Sung-Hye Park ${ }^{1,4,5^{*}}$

\begin{abstract}
Background: Tuberous sclerosis complex (TSC) is an autosomal dominant disorder characterized by hamartomas in multiple organs associated with germline mutations in TSC1 and TSC2, including exonic, intronic, or mosaic mutations. Gastrointestinal (GI) tract Lymphangioleiomyomatosis (LAM) is an extremely rare manifestation of TSC, with few reported cases. Herein, we aimed to determine the driver mutation, pathogenesis, and relationship of germline and somatic mutations of LAM through whole-genome sequencing (WGS) of the tumor and blood samples and whole transcriptome sequencing (WTS) analysis.
\end{abstract}

Case presentation: A nine-year-old girl with a full-blown TSC presented with abdominal masses detected during a routine check-up. Resected intestinal masses were diagnosed as LAM by thorough pathological examination. Interestingly, the LAM presented a somatic TSC2 gene mutation in exon 24 (p.R905W, c.C2713T), and the patient had intron retention by a novel germline mutation in the intron region of TSC2 (chr16:2126489, C > G).

Conclusion: Our case suggests that intron retention by a single nucleotide intronic mutation of TSC2 is sufficient to develop severe manifestations of TSC, but the development of LAM requires an additional somatic oncogenic mutation of TSC2.

Keywords: Intron retention, Lymphangioleiomyomatosis, Germline mutation, Somatic mutation, Perivascular epithelioid cell tumor (PEComa), Tuberous sclerosis complex

\footnotetext{
* Correspondence: hyunjulee@gist.ac.kr; shparknp@snu.ac.kr

${ }^{2}$ School of Electrical Engineering and Computer Science, Gwangju Institute

of Science and Technology, 123 Cheomdangwagi-ro, Buk-gu, Gwangju

61005, Republic of Korea

'Department of Pathology, Seoul National University Hospital, Seoul National

University College of Medicine, 103 Daehak-ro, Jongno-gu, Seoul 03080,

South Korea

Full list of author information is available at the end of the article
}

(C) The Author(s). 2021 Open Access This article is licensed under a Creative Commons Attribution 4.0 International License, which permits use, sharing, adaptation, distribution and reproduction in any medium or format, as long as you give appropriate credit to the original author(s) and the source, provide a link to the Creative Commons licence, and indicate if changes were made. The images or other third party material in this article are included in the article's Creative Commons licence, unless indicated otherwise in a credit line to the material. If material is not included in the article's Creative Commons licence and your intended use is not permitted by statutory regulation or exceeds the permitted use, you will need to obtain permission directly from the copyright holder. To view a copy of this licence, visit http://creativecommons.org/licenses/by/4.0/ The Creative Commons Public Domain Dedication waiver (http://creativecommons.org/publicdomain/zero/1.0/) applies to the data made available in this article, unless otherwise stated in a credit line to the data. 


\section{Background}

Herein, we report a case of Tuberous sclerosis complex (TSC) with hamartomas in multiple organs and gastrointestinal lymphangioleiomyomatosis (GI LAM). TSC is an autosomal dominant disorder characterized by hamartomas in multiple organs, seizure disorders, mental retardation, and a reported prevalence of $1 / 6000$ to 1/10,000 live births [1, 2]. TSC1 (9q34) and TSC2 (16p13), both known tumor suppressors, are the causative genes. Mutations in these genes result in dysregulation of the mammalian target of rapamycin (mTOR pathway) [3]. To date, more than 1000 mutations have been reported in TSC1 and TSC2 [4]. However, 10-15\% of clinically diagnosed TSC have no known identifiable mutations and could be attributed to mutations in the promoter, enhancer, or intron region, as well as mosaicism or technical problems [4-6]. Tyburczy et al. have identified mutations in $85 \%$ (45 of 53) of patients with TSC in whom no mutations were initially identified by conventional testing [6]. Most of these mutations can be attributed to mosaicism (58\%) and intronic mutations (40\%) [6]. TSC patients with mosaicism tend to present less severe symptoms, consistent with the gene dosage effect [5]. Intronic mutations are rare and remain undetected with whole-exome sequencing (WES) and with whole-genome sequencing (WGS), special analysis algorithms are still required.

Lymphangioleiomyomatosis (LAM) is a rare neoplasm. Notably, it is a type of perivascular epithelioid cell tumor (PEComa), characterized by aberrant proliferation of smooth muscle-like cells that express melanocytic (HMB45 and Melan-A) and smooth muscle markers (smooth muscle actin, desmin, and caldesmon), as well as melanocyte inducing transcription factor/ Microphthalmia-Associated Transcription Factor (MITF) [7]. There are two identified subtypes: sporadic-LAM (S-LAM) and TSC-related LAM (TSCLAM). Both are induced by TSC1 or, more commonly, TSC2 gene mutations [8]. The gastrointestinal (GI) tract is a rare site for LAM.

By employing WGS and transcriptome studies, we detected germline intronic and somatic TSC2 gene mutations in this patient. Typically, intron retention has been considered "noise" as it is technically challenging to detect and quantify transcripts with retained introns globally [9]. However, we hypothesize that intron retention of the TSC2 protein by intron mutation could be a driver mutation of TSC and that TSC2 somatic mutation is responsible for the development of LAM. The percentage of TSC1/TSC2 second-hit tumors is $65.1 \%$ of TSC-related tumors [10]. Herein, we focus on the tumorigenic mechanism of intron retention and second hit somatic mutation of TSC2 observed in LAM.

\section{Case presentation}

A nine-year-old girl presented with abdominal masses detected in an abdominal sonogram during follow-up check for her TSC manifestations and any tumor development.

Prenatal ultrasound first detected nodules in her heart. Under possibility of TSC, ophthalmology and brain MRI were performed after birth and revealed hamartomas in eyes and intraventricular nodules, suggesting subependymal giant cell astrocytoma (SEGA). She was diagnosed with TSC through genetic test conducted at outside hospital. Myoclonic seizures were observed when she was approximately 100 days old. Furthermore, she presented mental retardation and intermittent febrile or afebrile generalized tonic-clonic seizures, with an abnormal electroencephalogram. She was prescribed an antiepileptic drug and was under close observation. Abdominopelvic and chest computed tomography scans showed multiple small nodules in both kidneys, lungs, and intestines, from the cecum to the ascending colon (Fig. 1). She then underwent laparoscopy-assisted right hemicolectomy. No events related to GI LAM occurred during the 2year postoperative follow-up period.

\section{Pathology and immunohistochemistry}

On gross examination, multiple nodules were observed along the cecum wall, up to the ascending colon. The largest nodule was $6.1 \mathrm{~cm}$ (Fig. 2). The nodules were well-demarcated, tan-to-white, and had solid consistency, with hemorrhage and necrosis. Microscopically, the tumor was composed of smooth muscle-like spindle cells, with bland-looking elongated nuclei and eosinophilic cytoplasm arranged in a fascicular pattern. Tumor cells were distributed around the slit-like vascular spaces lined by flattened endothelial cells. Focal necrosis was observed in the center of the largest nodule, and the mitotic index was $2 / 50$ high power fields. In total, 4 out of the 17 lymph nodes presented metastatic LAM.

Immunohistochemical staining was performed on an immunostaining system (BenchMark ULTRA system, Ventana-Roche, Mannheim, Germany) using primary antibodies, including HMB45 (A: 200, Millipore, Temecula, US), D2-40 (1: 50, DAKO, Glostrup, Denmark), smooth muscle actin (SMA, 1:500, DAKO), c-kit (CD117, 1: 500, Abcam, Cambridge, UK), CD34 (1:200, DAKO), caldesmon (1: 1000, DAKO), desmin (1:200, DAKO), myogenin (1: 500, DAKO), Melan-A (1: 1000, Cell Marque, Rocklin, USA), and Ki-67 (1: 100, DAKO) (Table 1). Appropriate positive controls were included, and primary antibodies were omitted from negative controls.

The tumor cells expressed HMB45, D2-40, GLUT-1, and SMA but were negative for c-kit and CD34, both 

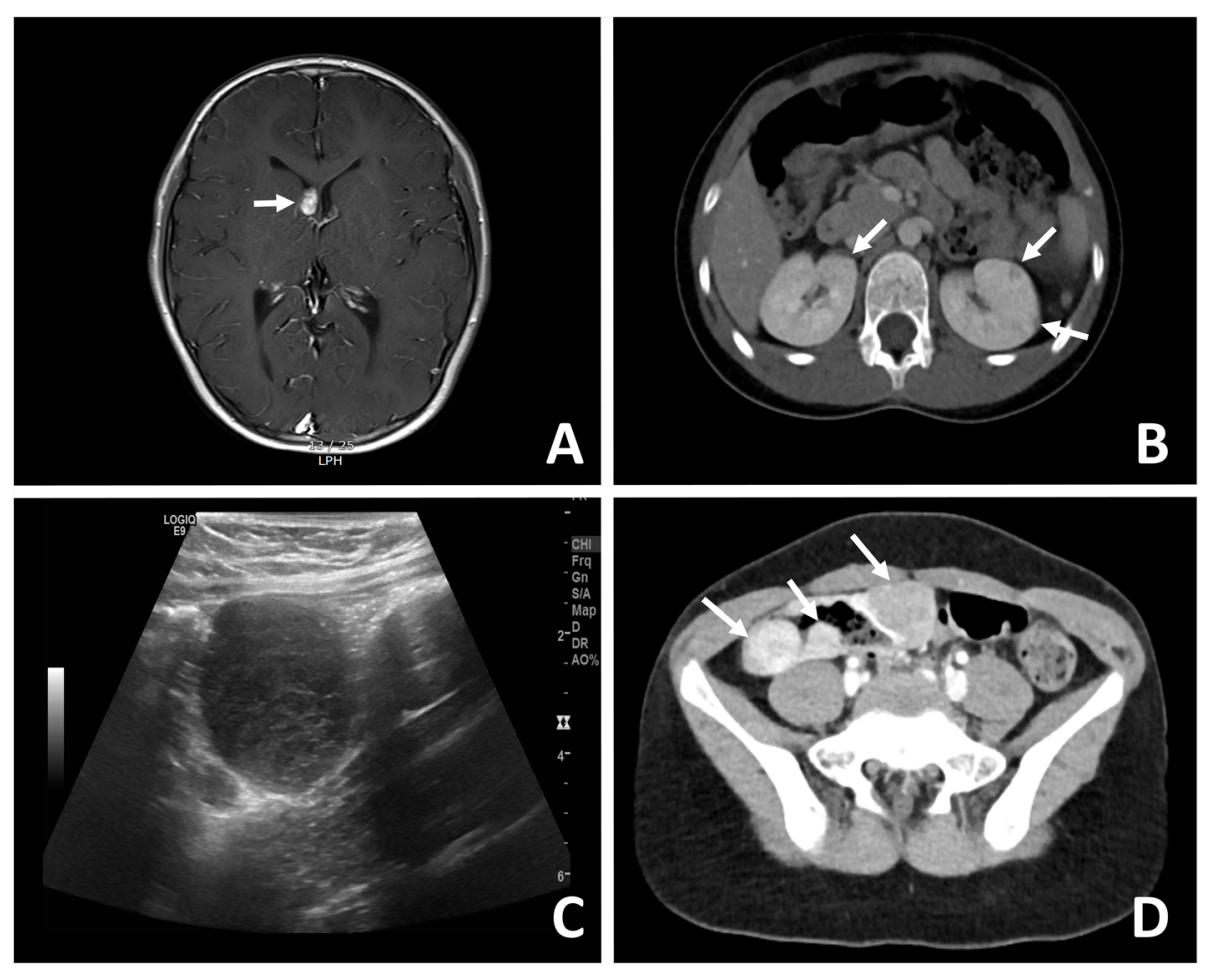

Fig. 1 A Brain MRI showing an ovoid, enhancing mass at the right lateral ventricle (arrow). B Abdominal CT scan showing multiple lowattenuated foci in both kidneys (arrows). C Ultrasonography findings showing multiple low echoic nodules along the bowel wall. D Abdominal CT scan showing multiple enhancing masses along the ascending colon wall (arrows). MRI, magnetic resonance imaging; $C T$, computed tomography

specific GI stromal tumor markers (GIST) (Fig. 2). Moreover, tumor cells were negative for caldesmon, desmin, myogenin, and Melan-A and S-100. We ruled out leiomyomatosis by HMB45 expression in our tumor and also excluded malignant melanoma by negative for Melan-A and S-100 in our tumor. The Ki-67 labeling index was $10.0 \%$. Immunostaining with D2-40 highlighted endothelial cells lining the slit-like channels. Hence, the lesion was diagnosed as a LAM.

\section{Preprocessing and analysis of WGS and WTS}

Representative tumor areas with at least 90\% tumor cell content were outlined for macrodissection on hematoxylin-eosin-stained fresh frozen tissue sections. DNA/RNA extraction was performed from the freshly frozen tissue and patient blood using the Maxwell ${ }^{\circ}$ RSC DNA/RNA FFPE Kit (Promega, USA).

WGS data were generated using the TruSeq DNA PCR Free library kit and Illumina platform, which had a $150 \mathrm{bp}$ read length, $408 \mathrm{bp}$ fragment length median, and $1,450,195,688$ total reads. The paired-end sequence was mapped to the human genome (original GRCh37 from NCBI, February 2009) using Isaac aligner (iSAAC04.18.11.09). The Isaac aligner identifies and selects the foremost mapping candidates using a 32-mer seed-based search. The 3 end with low quality and adapter sequences were trimmed from the alignment. The Isaac aligner generates a binary alignment output bam file that includes sorted and duplicate-marked data.

WTS data were produced using the SureSelectXT RNA Direct library kit and NovaSeq 6000 platform, creating a $101 \mathrm{bp}$ read length and 161,213,862 total reads. The STAR aligner was used for RNA-Seq data to create a sorted bam file, and its index was created using Samtools v1.9. RSEM [11], with edgeR [12] steps performed to obtain the expression values. The fastq files of the WGS and WTS samples had high Phred quality scores. For tumor WGS, normal blood WGS, and tumor WTS samples, 97.2, 97.6, and $98.42 \%$ of the bases had quality scores $>20$ for tumor WGS, normal WGS, and tumor WTS samples, respectively.

\section{Detection of somatic mutation}

Somatic mutations for single nucleotide variants (SNVs) and insertions and deletions (INDELs) were detected using the Genome Analysis Toolkit (GATK) Mutect2 v4.1.4.1 [13] with default parameters. All the variants were annotated by ANNOVAR (https://doc-openbio. readthedocs.io/projects/annovar/en/latest/) [14]. 

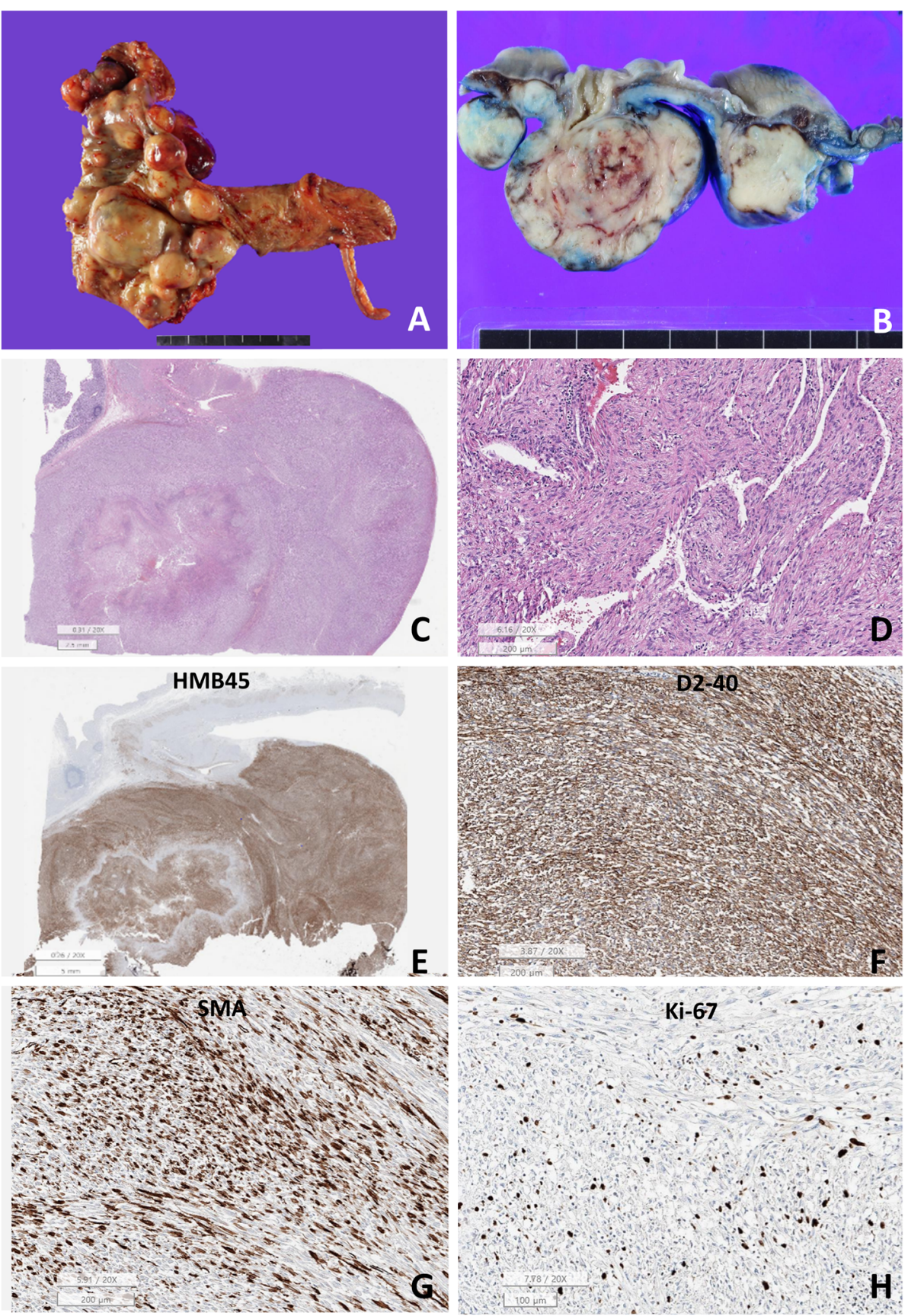

Fig. 2 A The tumor is well-demarcated, with masses along the intestinal wall. B, C On sectioning and low power view, the tumor appears to replace the proper muscle layer of the cecum with central suppurative necrosis. D High power view of the tumor shows spindle shape mesenchymal cells with slit-like lymphatic channels. The tumor cells show little nuclear atypia. E, F The tumor cells are positive for HMB45 and D2-40. G Tumor is focally positive for smooth muscle actin (SMA). H Ki-67 index is 7.0\%. Above mentioned pathological and

immunohistochemical features are consistent with LAM. (A, B: gross picture, C, D: H\&E, E: HMB45, F: D2-40, G: SMA, H: Ki-67, lower bar: C=2.5 $\mathrm{mm}, \mathrm{D}=200 \mu \mathrm{m}, \mathrm{E}=5 \mathrm{~mm}, \mathrm{~F}, \mathrm{G}: 200 \mu \mathrm{m}, \mathrm{H}=100 \mu \mathrm{m})$. LAM, lymphangioleiomyomatosis

By employing the WGS data, we detected 12 genes with nonsynonymous somatic mutations and but only 2 mutations (MST1: p.E261Q, and TSC2: p.R905W) are pathogenic (Supplementary Table 1). Among them, TSC2 had a somatic hotspot mutation (p.R905W,
c.C2713T) (Fig. 3A). We assessed whether this deleterious variant in TSC2 was expressed in mRNA using RNA-Seq data from the tumor sample. STAR 2-PASS [15] was used to create the bam file from the RNA-Seq data, and the reads were visualized using IGV [16]. In 
Table 1 Primary antibodies used in this study

\begin{tabular}{llll}
\hline Antibody & Dilution & Antigen retrival & Source \\
\hline HMB45 & $1: 200$ & Ventana CC1 $100^{\circ} \mathrm{C}$ & Milipore, Temecula, US \\
Melan-A (Mart-1) & $1: 1000$ & Ventana CC1 $100^{\circ} \mathrm{C}$ & Cell Marque, Rocklin, US \\
D2-40 & $1: 50$ & Ventana CC1 $100^{\circ} \mathrm{C}$ & DAKO, Glostrup, Denmark \\
Phosphorylated mTOR & $1: 100$ & Ventana CC1 $100^{\circ} \mathrm{C}$ & Cell signaling, Boston, US \\
C-Kit & $1: 500$ & Ventana CC1 $100^{\circ} \mathrm{C}$ & Abcam, Cambridge, UK \\
SMA & $1: 500$ & Ventana CC1 $100^{\circ} \mathrm{C}$ & DAKO, Glostrup, Denmark \\
CD34 & $1: 200$ & Ventaan CC1 $100^{\circ} \mathrm{C}$ & DAKO, Glostrup, Denmark \\
Caldesmon & $1: 1000$ & Ventaan CC1 $100^{\circ} \mathrm{C}$ & DAKO, Glostrup, Denmark \\
Desmin & $1: 200$ & Ventaan CC1 $100^{\circ} \mathrm{C}$ & DAKO, Glostrup, Denmark \\
Myogenin & $1: 500$ & Ventaan CC1 $100^{\circ} \mathrm{C}$ & DAKO, Glostrup, Denmark \\
Ki-67 & $1: 100$ & Ventana CC1 $100^{\circ} \mathrm{C}$ & DAKO, Glostrup, Denmark \\
\hline
\end{tabular}

total, 570 reads were mapped in the concerned region, and 390 (variant allele frequency [VAF]: 68.0\%) had variant sequences.

\section{Detection of germline mutation}

GATK Haplotypecaller [17] was used to identify germline mutations from the WGS bam of normal data. To ensure a high confidence germline mutation, we performed a VariantRecalibrator step of GATK and used the vcf files including dbsnp_138.hg19.vcf [18], hapmap_ 3.3.hg19.sites.vcf [19], 1000G_omni2.5.hg19.sites.vcf [20], 1000G_phase1.snps.high_confidence.hg19.sites.vcf [21], and Mills_and_1000G_gold_standard.indels.hg19.sites.vcf [22], which were obtained from the GATK resource bundle.

In the present patient with TSC, we detected a novel TSC2 germline mutation in the TSC2 intron region, next to the acceptor site (chr16:2126489, C > G). This variant had not been previously reported in ClinVar [23]. We identified that 207 out of 216 reads (VAF: 96\%) were mapped to this germline mutation site.

A previously reported mutation at the same intron but into a different nucleotide (NM_000548.4 (TSC2): c.2743-3C > A) was observed in assembly GRCh37 (https://www.ncbi.nlm.nih.gov/clinvar/23291215/).

As reads in the RNA-Seq data were mapped to the intron region, iREAD and IRFinder tools were used to detect intron retention. By employing these two tools, intron retention was detected in the chr16:2126258$2,126,491$ region, where the germline mutation occurred (Fig. 3B). For comparison, we downloaded the RNA-Seq data of patients with sarcoma from The Cancer Genome Atlas (TCGA) to confirm that this event did not occur randomly. No intron retention was detected in the chr16:2126258-2,126,491 region on analyzing 256 samples with IRFinder (Fig. 3B). We postulate that this

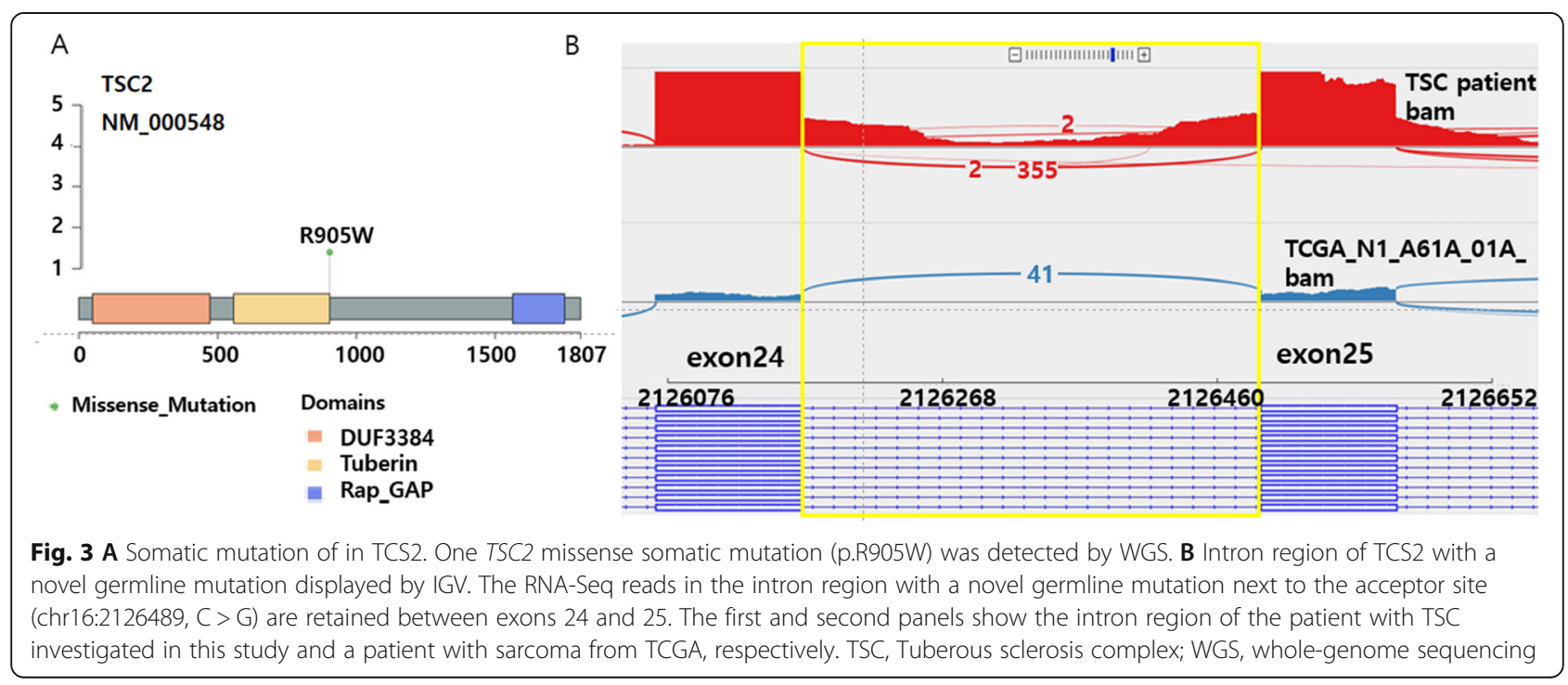


intron retention did not appear randomly and that the nearby acceptor site germline mutation might affect the mRNA splicing mechanism.

\section{Detection of intron retention}

iREAD [22] and IRFinder [24] were used to identify intron retention from RNA-Seq fastq data. We used the default parameters for the two tools, except for the entropy score of iREAD. The default threshold was strictly set in the iREAD package. We lowered the entropy score marginally, from 0.9 to 0.85 , to detect more candidate IR.

We obtained the trimmed mean of M-values (TMM) of genes with edgeR. The TMM expression value of the TSC2 gene was 28,576, which was 7.4-fold higher than the average of two standard samples (TMM: 3857).

For reverse transcriptase-polymerase chain reaction (RT-PCR), RNA was extracted from the peripheral blood buffy coat and tumor (lymphangioleiomyoma) of this patient and the peripheral blood buffy coat of two healthy (negative) controls. Primer sets were designed to detect TSC2 gene-splicing region in the intron between the exon 24 and exon 25 (Forward primer: GTCATAGCCA TGTGGTTCA, Reverse primer: TCTTGGGTCTCTCG TTGA). RT-PCR of this patient's peripheral blood and tumor RNA revealed the intron retention products at
$530 \mathrm{bp}$ and splicing products at $163 \mathrm{bp}$, but healthy controls showed splicing products at 163 bp only (Fig. 4).

\section{Discussion and conclusions}

TSC is an autosomal dominant disorder caused by genetic alterations in TSC1 at 9p34 (22\%) and TSC2 at $16 \mathrm{p} 13$ (63\%), with no mutation identified in about $15 \%$ of patients [3]. TSC1 and TSC2 encode two tumor suppressor proteins: hamartin and tuberin. Inactivating mutations in either gene fail to inhibit the mTOR pathway and lead to constitutive activation of mTORC1 and dysregulation of downstream molecules, driving uncontrolled cell growth and proliferation; this results in the formation of either hamartomas or benign tumors in multiple organs, including the skin, eyes, heart, kidneys, and brain $[3,7,25]$.

LAM is a rare neoplasm that belongs to the family of PEComas [26]. Notably, LAM is six times more common in females than males, and the average age of patients is 42.4 years. The relationship between TSC and LAM is well established. Pulmonary LAM and renal angiomyolipomas are common manifestations of TSC, occurring in 80 and $70 \%$ of TSC patients, respectively [1,27]. Somatic mutations have been detected in sporadic LAMs (https://databases.lovd.nl/shared/diseases/00317).

In patients with TSC, GI manifestations of LAM are infrequent, and only two cases have been reported [28,

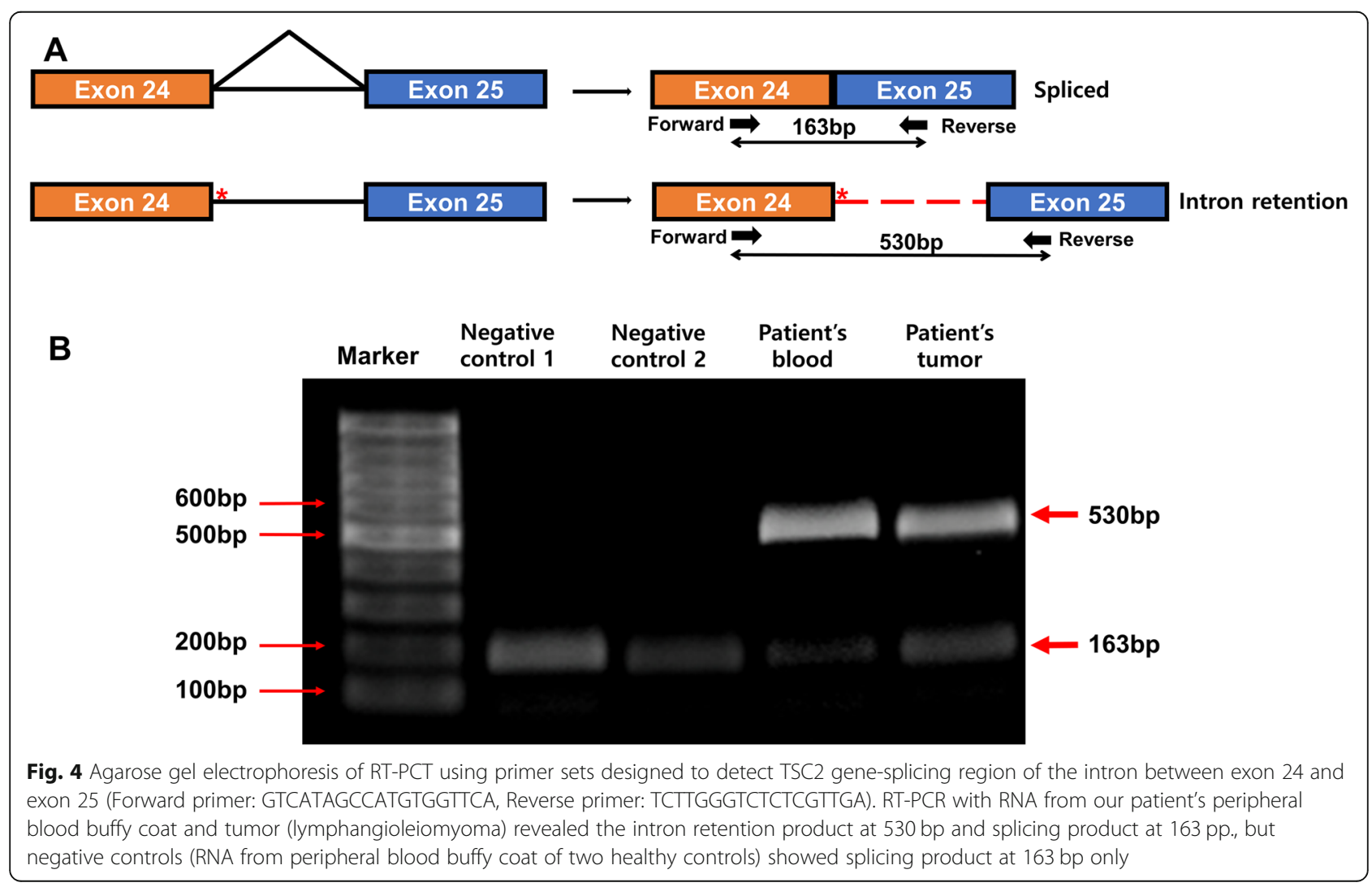


29]. One patient had a germline frameshift mutation in TSC2 (p.K69Nfs*37). The presence of somatic mutations has not been investigated in the remaining patients.

The diagnosis of LAM remains challenging owing to its rarity in extrapulmonary sites [28, 29]. Leiomyomatosis, GIST, and melanoma are the differentials in the present case. Both LAM and leiomyomatosis present as multiple nodules along the bowel walls and show smooth muscle cell proliferation with the expression of smooth muscle markers. GIST comprises spindle and epithelial cells, with c-kit, CD34, or Dog1 expressions. Notably, the melanocytic marker, HMB-45, helps distinguish LAM from leiomyomatosis and GIST, as LAM is $100 \%$ immunoreactive for HMB-45, whereas the other two entities are not [30].

In women with TSC, the prevalence of TSC-LAM is $34 \%$; however, the estimated prevalence of S-LAM is approximately 10,000 patients worldwide, which is considerably less than that of TSC-LAM [31, 32]. Both S-LAM and TSC-LAM are associated with mutations in TSC1 and, more commonly, in TSC2 genes [33]. Through WGS, we identified a somatic gene mutation, p.R905W (c.C2713T), in the TSC2 region (of chromosome 16p13). Although this variant has been reported in many individuals with TSC, functional and experimental studies, as well as computational prediction algorithm studies included in ANNOVAR [14], have shown that this mutation is pathogenic as it affects protein function [34]. Arginine at codon 905 is a critical amino acid for the function of tuberin, and two missense mutations, 2714G > A R905Q and 2713C > T R905W in TSC2, have been reported at this codon. Both variations influence the mTOR signaling pathway, affecting cell proliferation, migration, survival, and metabolism [7]. Alteration into different amino acids in the same codon results in varying severity among phenotypes. The R905Q mutation was detected in TSC families with a relatively mild phenotype (normal cognition with no SEGA or epilepsy) [35]. The R905W mutation was found in sporadic TSC and manifested with severe symptoms. According to an article by Sancak et al, all six patients with the R905W mutation presented cortical tubers and SEGA, cognitive impairment, neurologic symptoms, and additional TSC features [36]. Our patient showed a somatic missense mutation (R905W) in LAM and a novel germline intronic mutation (chr16:2126489, C > G), with severe neurological symptoms and multi-organ hamartomas. A known intronic mutation at the same site changed to a different nucleotide (NM_000548.5: c.2743-3C > A, https://www.ncbi.nlm.nih.gov/clinvar/ variation/49229/), which affects gene splicing. This variant is not present in population germline databases. When we performed splice site prediction using "Berkeley Drosophila Genome Project, splice site prediction by neural network" [37], this variant was predicted to affect normal splice donor site with the 0.99 prediction score. The novel germline intron mutation found in our case is considered a pathogenic variant with moderate evidence of pathogenicity in TSC.

We provided both RNAseq and RT-PCR confirmation of intron retention. It is justified that the two different methods for the detection of intron retention are consistent. Of note, RNA analysis does not necessarily demonstrate loss of normal TSC2 function, which negatively regulates mTOR signaling. However, intron retention has the potential to cause loss of function, consistent with disease mechanisms.

Recent studies have reported that approximately 20\% of PEComas also have a transcription factor E3 (TFE3) mutation. PEComas harboring TFE3 gene rearrangement have been previously reported, presenting an epithelioid appearance, weak or no expression of smooth muscle markers, and robust nuclear staining of TFE3 protein $[38,39]$. In the present case, TFE3 rearrangement was not observed. Moreover, TFE3 rearrangements and TSC2 mutations are known to be mutually exclusive [40]. Cutaneous PEComas do not harbor TFE3 rearrangements, and the occurrence of TFE3 rearrangement might vary among organs that develop tumors [41].

A novel intron heterozygous mutation in TSC2 (chr16: 2126489, C > G) was detected through WGS and RNA sequencing. Intron retention is an alternative splicing method [42] and plays a regulatory role in neuronal differentiation and neurological diseases [43]. Two different intron retentions of TSC2 have been reported in patients with TSC (chr16:2106052, C > T and chr16:2126489, C > A) [44, 45]. Different mutations at the same acceptor site of intron have been reported as possibly pathogenic (chr16:2126483,C > G) [46]. Intron retention by intronic mutation of TSC2 might contribute to our patient's TSC pathogenesis with multiple hamartomas; however, it remains unclear whether the germline mutation of TSC2 is pathogenic, given that TSC2 was overexpressed. As TSC2 is a tumor suppressor, it needs to be underexpressed to be considered a pathogenic mutation. Therefore, further evaluation of the potential biological effects of intron retention in TSC is required.

GI PEComas show a variable spectrum of biological behavior. Folpe et al. have reported that clinically malignant PEComas typically have infiltrative growth patterns, large tumor size $(>5 \mathrm{~cm})$, high nuclear grade, tumor necrosis, increased mitotic activity ( $>1 / 50 \mathrm{HPF})$, and lymphovascular invasion [30]. A recent study regarding GI PEComas has reported that this tumor demonstrates malignant behavior significantly associated with marked nuclear atypia, diffuse pleomorphism, and mitosis $\geq 2 / 10$ HPF [47]. The present patient presented a tumor exceeding $5 \mathrm{~cm}$ in size, infiltrative growth, tumor necrosis, 
a mitotic index of 2/50 HPF, and a Ki-67 labeling index of $10 \%$, consistent with malignancy. Additionally, lymph node metastasis was detected. Previous reports have suggested that surgical resection is the best treatment strategy. Considering the genetic etiology of LAM affecting the mTOR pathway, mTOR inhibitors can be a treatment of choice. The mTOR inhibitor rapamycin has several advantages in patients with LAM, stabilizing lung function and improving quality of life. However, the discontinuation of therapy results in disease progression $[7$, 48].

In conclusion, we presented a case of a TSC patient with extrapulmonary LAM in addition to classic TSC triads. We identified a novel TSC2 germline intronic mutation (chr16:2126489, C>G) and a second hit somatic TSC2-mutation in exon 24 (p.R905W, c.C2713T) in the patient's blood and tumor samples. LAM diagnosis and treatment can be challenging; nevertheless, it is essential to be aware of the association between TSC $2 \mathrm{mu}$ tations and LAM. Our findings provide clues for the repair and prevention of TSC and suggest that intron retention by intronic mutation of TSC 2 is sufficient to develop severe TSC manifestations; however, the development of LAM requires an additional somatic oncogenic mutation. Future multi-omics studies need to elucidate the precise pathogenesis and tumorigenesis of TSC and establish a suitable treatment strategy for this disease.

\section{Abbreviations}

CT: Computed tomography; GIST: Gastrointestinal stromal tumor markers; LAM: Lymphangioleiomyomatosis; MITF: Melanocyte inducing transcription factor/microphthalmia transcription factor; MRl: Magnetic resonance imaging; PEComa: Perivascular epithelioid cell tumor; SEGA: Subependymal giant cell astrocytoma; TSC: Tuberous sclerosis complex; WGS: Whole-genome sequencing; WTS: Whole transcriptome sequencing

\section{Supplementary Information}

The online version contains supplementary material available at https://doi. org/10.1186/s13000-021-01138-8

\section{Additional file 1: Supplementary Table 1. Somatic}

nonsynonymous single nucleotide variation detected by WGS in our lymphangioleiomyoma, but most were VUS except MST1 and TSC2.

\section{Acknowledgements}

This work was supported by IITP grant funded by the Korean government (MSIP) (No.2019-0567).

\section{Authors' contributions}

Bogyeong Han reviewed the case and wrote the manuscript, Hyun-Young Kim carried out operation and patient care, Juhwan Lee, Hyunju Lee, Yumi Shim, Kwang Hoon Lee preperated and analysed NGS data, Yoon Jin Kwak reviewed the pathology, and Sung-Hye Park supervised this research and edited the manuscript. The authors read and approved the final manuscript.

\section{Funding}

This work was supported by the Institute for Information \& Communications Technology Promotion (IITP) grant funded by the Korean government (MSIP) (No.2019-0567, Development of Intelligent SW systems for uncovering genetic variation and developing personalized medicine for cancer patients with unknown molecular genetic mechanisms).

\section{Availability of data and materials}

The data that support the findings of this study are available from the corresponding authors (Hyunju Lee and Sung-Hye Park) upon reasonable request.

\section{Declarations}

Ethics approval and consent to participate

This study was approved by the Institutional Review Board of Seoul National University Hospital (IRB No: 1905-108-1035). All experiments were performed in accordance with the guidelines and regulations of the Helsinki and Human Research Protection Program.

\section{Consent for publication}

Since the patient suffers from mental retardation, informed consent was obtained from her father for the donation of the patient's human body materials. Since this study was a retrospective review of medical records, use donated bank samples and only analysis of disease-related alteration, informed consent for publication was waivered by IRB and in accordance with the Korean Bioethics Act and Biosafety Act. Personal identification was anonymized by the tissue bank and the authors never contact the patient or parents.

\section{Competing interests}

The authors do not have any conflicts of interest to declare.

\section{Author details}

'Department of Pathology, Seoul National University Hospital, Seoul National University College of Medicine, 103 Daehak-ro, Jongno-gu, Seoul 03080, South Korea. ${ }^{2}$ School of Electrical Engineering and Computer Science, Gwangju Institute of Science and Technology, 123 Cheomdangwagi-ro, Buk-gu, Gwangju 61005, Republic of Korea. 'Department of Surgery, Seoul National University Hospital (SNUH), Seoul National University College of Medicine, Seoul, Republic of Korea. ${ }^{4}$ Center for Medical Innovation, Seoul National University Hospital, Seoul, Republic of Korea. ${ }^{5}$ Department of Pathology, Seoul National University College of Medicine, Seoul 03080, Republic of Korea.

Received: 11 March 2021 Accepted: 11 August 2021

Published online: 31 August 2021

References

1. Carsillo T, Astrinidis A, Henske EP. Mutations in the tuberous sclerosis complex gene TSC2 are a cause of sporadic pulmonary lymphangioleiomyomatosis. Proc Natl Acad Sci. 2000;97:6085-90.

2. O'Callaghan FJ, Shiell AW, Osborne JP, Martyn CN. Prevalence of tuberous sclerosis estimated by capture-recapture analysis. Lancet. 1998;351:1490.

3. Fohlen $\mathrm{M}$, et al. Identification of TSC1 or TSC2 mutation limited to the tumor in three cases of solitary subependymal giant cell astrocytoma using next-generation sequencing technology. Childs Nerv Syst. 2020;36:961-5. https://doi.org/10.1007/s00381-020-04551-4.

4. Gao S, Wang Z, Xie Y. Two novel TSC2 mutations in pediatric patients with tuberous sclerosis complex: case report. Medicine (Baltimore). 2018;97: e11533. https://doi.org/10.1097/MD.0000000000011533.

5. Qin W, et al. Ultra deep sequencing detects a low rate of mosaic mutations in tuberous sclerosis complex. Hum Genet. 2010;127:573-82. https://doi. org/10.1007/s00439-010-0801-z

6. Tyburczy ME, et al. Mosaic and Intronic mutations in TSC1/TSC2 explain the majority of TSC patients with no mutation identified by conventional testing. PLoS Genet. 2015;11:e1005637. https://doi.org/10.1371/journal. pgen.1005637.

7. Moir LM. Lymphangioleiomyomatosis: current understanding and potential treatments. Pharmacol Ther. 2016;158:114-24.

8. Jonjic N, Roganovic J, Glavan N, Hadzisejdic I, Seili-Bekafigo I. Malignant perivascular Epitheloid cell tumor with an unusual Immunophenotype in a ten-year-old child. Fetal Pediatr Pathol. 2019:38:503-10. https://doi.org/10.1 080/15513815.2019.1604923. 
9. Jacob AG, Smith CWJ. Intron retention as a component of regulated gene expression programs. Hum Genet. 2017;136:1043-57. https://doi.org/10.1 007/s00439-017-1791-x.

10. Martin KR, et al. The genomic landscape of tuberous sclerosis complex. Nat Commun. 2017;8:15816. https://doi.org/10.1038/ncomms15816.

11. Li B, Dewey CN. RSEM: accurate transcript quantification from RNA-Seq data with or without a reference genome. BMC Bioinformatics. 2011;12:323. https://doi.org/10.1186/1471-2105-12-323.

12. Robinson MD, McCarthy DJ, Smyth GK. edgeR: a bioconductor package for differential expression analysis of digital gene expression data. Bioinformatics. 2010;26:139-40. https:/doi.org/10.1093/bioinformatics/btp616.

13. Benjamin $D$, et al. Calling somatic snvs and indels with mutect2. BioRxiv. 2019:861054. https://doi.org/10.1101/861054.

14. Wang K, Li M, Hakonarson H. ANNOVAR: functional annotation of genetic variants from high-throughput sequencing data. Nucleic Acids Res. 2010;38: e164. https://doi.org/10.1093/nar/gkq603.

15. Dobin A, et al. STAR: ultrafast universal RNA-seq aligner. Bioinformatics, 2013;29:15-21. https://doi.org/10.1093/bioinformatics/bts635.

16. Robinson JT, et al. Integrative genomics viewer. Nat Biotechnol. 2011;29:246. https://doi.org/10.1038/nbt.1754.

17. McKenna A, et al. The genome analysis toolkit: a MapReduce framework for analyzing next-generation DNA sequencing data. Genome Res. 2010;20: 1297-303. https://doi.org/10.1101/gr.107524.110.

18. Sherry ST, et al. dbSNP: the NCBI database of genetic variation. Nucleic Acids Res. 2001;29:308-11. https://doi.org/10.1093/nar/29.1.308.

19. International HapMap, C, et al. Integrating common and rare genetic variation in diverse human populations. Nature. 2010;467:52-8. https://doi. org/10.1038/nature09298.

20. Genomes Project C, et al. A map of human genome variation from population-scale sequencing. Nature. 2010;467:1061-73. https://doi.org/10.1 038/nature09534.

21. Mills RE, et al. An initial map of insertion and deletion (INDEL) variation in the human genome. Genome Res. 2006;16:1182-90. https://doi.org/10.1101/ gr.4565806.

22. Li HD, Funk CC, Price ND. iREAD: a tool for intron retention detection from RNA-seq data. BMC Genomics. 2020;21:128. https://doi.org/10.1186/s12864020-6541-0.

23. Landrum MJ, et al. ClinVar: public archive of relationships among sequence variation and human phenotype. Nucleic Acids Res. 2014;42:D980-5. https:// doi.org/10.1093/nar/gkt1113.

24. Middleton $R$, et al. IRFinder: assessing the impact of intron retention on mammalian gene expression. Genome Biol. 2017;18:51. https://doi.org/10.11 86/s13059-017-1184-4

25. Abdelwahed $M$, et al. A novel de novo splicing mutation C.1444-2A>T in the TSC2 gene causes exon skipping and premature termination in a patient with tuberous sclerosis syndrome. IUBMB Life. 2019;71:1937-45. https://doi.org/10.1002/iub.2134.

26. Matsui $K$, et al. Extrapulmonary lymphangioleiomyomatosis (LAM): clinicopathologic features in 22 cases. Hum Pathol. 2000:31:1242-8.

27. Northrup $\mathrm{H}$, et al. Tuberous sclerosis complex diagnostic criteria update: recommendations of the 2012 international tuberous sclerosis complex consensus conference. Pediatr Neurol. 2013;49:243-54.

28. Goh SGN, et al. Leiomyomatosis-like lymphangioleiomyomatosis of the colon in a female with tuberous sclerosis. Mod Pathol. 2001;14:1141-6.

29. Kolin DL, et al. Expanding the Spectrum of colonic manifestations in tuberous sclerosis: L-cell neuroendocrine tumor arising in the background of rectal PEComa. Endocr Pathol. 2018;29:21-6.

30. Folpe $\mathrm{AL}$, et al. Perivascular epithelioid cell neoplasms of soft tissue and gynecologic origin: a clinicopathologic study of 26 cases and review of the literature. Am J Surg Pathol. 2005;29:1558-75. https://doi.org/10.1097/01.pas. 0000173232.22117.37.

31. Harknett $\mathrm{E}$, et al. Use of variability in national and regional data to estimate the prevalence of lymphangioleiomyomatosis. QJM: Int J Med. 2011;104:971-9.

32. Moss J, et al. Prevalence and clinical characteristics of lymphangioleiomyomatosis (LAM) in patients with tuberous sclerosis complex. Am J Respir Crit Care Med. 2001;164:669-71.

33. Liu Y, et al. Lymphangioleiomyomatosis: a case report and review of diagnosis and treatment. OncoTargets Ther. 2018;11:5339.

34. Hoogeveen-Westerveld $\mathrm{M}$, et al. Functional assessment of variants in the TSC1 and TSC2 genes identified in individuals with tuberous sclerosis complex. Hum Mutat. 2011;32(4):424-35.
35. Jansen AC, et al. Unusually mild tuberous sclerosis phenotype is associated with TSC2 R905Q mutation. Ann Neurol. 2006;60:528-39. https://doi.org/10.1 002/ana.21037.

36. Sancak O. Tuberous sclerosis complex: mutations, functions and phenotypes; 2005.

37. Reese MG, Eeckman FH, Kulp D, Haussler D. Improved splice site detection in genie. J Comput Biol. 1997;4:311-23. https://doi.org/10.1089/cmb.1 997.4.311.

38. Agaram NP, et al. Dichotomy of genetic abnormalities in PEComas with therapeutic implications. Am J Surg Pathol. 2015;39:813.

39. Argani $P$, et al. A distinctive subset of PEComas harbors TFE3 gene fusions. Am J Surg Pathol. 2010;34:1395-406.

40. Malinowska I, et al. Perivascular epithelioid cell tumors (PEComas) harboring TFE3 gene rearrangements lack the TSC2 alterations characteristic of conventional PEComas: further evidence for a biologic distinction. Am J Surg Pathol. 2012;36:783

41. Llamas-Velasco M, et al. Cutaneous PEC oma does not harbour TFE 3 gene fusions: immunohistochemical and molecular study of 17 cases. Histopathology. 2013;63:122-9.

42. Broseus $L$, Ritchie W. Challenges in detecting and quantifying intron retention from next generation sequencing data. Comput Struct Biotechnol J. 2020;18:501-8. https://doi.org/10.1016/j.csbj.2020.02.010.

43. Mauger $\mathrm{O}$, Lemoine $\mathrm{F}$, Scheiffele $\mathrm{P}$. Targeted intron retention and excision for rapid gene regulation in response to neuronal activity. Neuron. 2016;92: 1266-78. https://doi.org/10.1016/j.neuron.2016.11.032.

44. Ye $Y$, Zeng $Y$. Whole exome sequencing identifies a novel intron heterozygous mutation in TSC2 responsible for tuberous sclerosis complex. Sci Rep. 2019;9:4456. https://doi.org/10.1038/s41598-019-38898-9.

45. Information., N. C. f. B. ClinVar; [VCV000049229.4], https://www.ncbi.nIm.nih. gov/clinvar/variation/NCV000049229.4. (2020).

46. Information., N. C. f. B. ClinVar; [VCV000065300.3], https://www.ncbi.nlm.nih. gov/clinvar/variation/65300/ (2021)

47. Doyle LA, Hornick JL, Fletcher CD. PEComa of the gastrointestinal tract: clinicopathologic study of 35 cases with evaluation of prognostic parameters. Am J Surg Pathol. 2013:37:1769-82.

48. Mohammadieh AM, Bowler SD, Silverstone EJ, Glanville AR, Yates DH. Everolimus treatment of abdominal lymphangioleiomyoma in five women with sporadic lymphangioleiomyomatosis. Med J Aust. 2013;199:121-3. https://doi.org/10.5694/mja12.11567.

\section{Publisher's Note}

Springer Nature remains neutral with regard to jurisdictional claims in published maps and institutional affiliations.

Ready to submit your research? Choose BMC and benefit from:

- fast, convenient online submission

- thorough peer review by experienced researchers in your field

- rapid publication on acceptance

- support for research data, including large and complex data types

- gold Open Access which fosters wider collaboration and increased citations

- maximum visibility for your research: over $100 \mathrm{M}$ website views per year

At $\mathrm{BMC}$, research is always in progress.

Learn more biomedcentral.com/submissions 